Purdue University

Purdue e-Pubs

2008

\title{
Simulation of Thermal Transport in Open-Cell Metal Foams: Effect of Periodic Unit Cell Structure
}

\author{
S. Krishnan \\ Purdue University \\ S V. Garimella \\ Purdue University, sureshg@purdue.edu \\ J.Y. Murthy \\ Purdue University
}

Follow this and additional works at: http://docs.lib.purdue.edu/coolingpubs

Krishnan, S.; Garimella, S V.; and Murthy, J. Y., "Simulation of Thermal Transport in Open-Cell Metal Foams: Effect of Periodic Unit Cell Structure" (2008). CTRC Research Publications. Paper 263.

http://dx.doi.org/10.1115/1.2789718

This document has been made available through Purdue e-Pubs, a service of the Purdue University Libraries. Please contact epubs@purdue.edu for additional information. 


\section{Simulation of Thermal Transport in Open-Cell Metal Foams: Effect of Periodic Unit-Cell Structure}

\section{Shankar Krishnan ${ }^{1}$}

\author{
Suresh V. Garimella \\ e-mail: sureshg@ecn.purdue.edu
}

\section{Jayathi Y. Murthy}

Cooling Technologies Research Center, School of Mechanical Engineering, Purdue University, West Lafayette, IN 47907-2088

Direct simulation of thermal transport in open-cell metal foams is conducted using different periodic unit-cell geometries. The periodic unit-cell structures are constructed by assuming the pore space to be spherical and subtracting the pore space from a unit cube of the metal. Different types of packing arrangement for spheres are considered-body centered cubic, face centered cubic, and the A15 lattice (similar to a Weaire-Phelan unit cell)-which give rise to different foam structures. Effective thermal conductivity, pressure drop, and Nusselt number are computed by imposing periodic boundary conditions for aluminum foams saturated with air or water. The computed values compare well with existing experimental measurements and semiempirical models for porosities greater than $80 \%$. The effect of different foam packing arrangements on the computed thermal and fluid flow characteristics is discussed. The capabilities and limitations of the present approach are identified. [DOI: 10.1115/1.2789718]

\section{Introduction}

Foams and other highly porous cellular solids have many interesting combinations of physical, mechanical, electrical, and thermal properties such as high stiffness, flow permeability, and thermal and electrical conductivity along with low specific weight. Many common materials are highly porous in nature. Examples of such highly porous natural materials include cork, wood, bones (trabecular and cancellous), and sponges [1-3]. Current state-ofthe-art manufacturing methods allow foaming of polymers, most metals and their alloys, ceramics, and graphite. Among the available synthetic open porous materials, polymer foams have enjoyed widespread use in many industrial technologies [3]. Recently, open-cell metal foams have been attracting increased attention as multifunctional materials due to their versatility in absorbing sound and energy [2]. Open-cell foams have a random reticulated structure of open polyhedral cells connected by continuous metal ligaments. Due to their highly porous reticulated nature, open-cell foams with negative Poisson's ratio (termed auxetic foams) have been produced by Lakes [4] and by many others [5] from conventional positive Poisson's ratio materials. Depending on the porosity of the cellular materials, a wide range of applications is possible. Figure 1 shows the variation of the degree of openness of the foams and their corresponding application [3].

\footnotetext{
${ }^{1}$ Currently at Bell Labs Ireland, Dublin, Ireland.

Contributed by the Heat Transfer Division of ASME for publication in the JouRNAL OF HeAT TRANSFER. Manuscript received December 8, 2006; final manuscrip received June 4, 2007; published February 6, 2008. Review conducted by Jamal Seyed-Yagoobi. Paper presented at the 2006 ASME International Mechanical Engineering Congress (IMECE2006), Chicago, IL, November 5-10, 2006.
}

Open-cell foams have been proposed for many applications such as porous tissue engineering scaffolds [6,7], hydrogen storage technologies [8], thermal control of electronics, catalysis [9], solar energy storage [10], electrochemical cells [11], and others $[2,12]$. The present study is primarily concerned with thermal management applications. With increased heat dissipation requirements for microprocessors [13] and many other electronics systems, demand for the development of compact heat dissipation systems continues to increase. Open-cell materials, owing to their ability to integrate multiple functions, are very attractive as thermal management materials. Many previous investigators have proposed and analyzed metal foams for possible applications as heat exchangers [14-16], heat sinks $[17,18]$, and heat pipe wicks.

Experimental measurements and numerical modeling to understand the overall, macroscopic thermal and fluid flow characteristics such as effective thermal conductivity, friction factor, and Nusselt number have been conducted by many previous investigators [15,19-21]. However, not many studies have attempted a detailed understanding at the pore scale. Some of the available literature on the microscopic approach to modeling foams is reviewed in Ref. [22]. Detailed modeling of pore-scale heat transfer is necessary for a number of reasons. Resolution of flow and heat transfer at the pore scale has been found to yield accurate predictions of effective thermal conductivity, pressure drop, and local heat transfer coefficient. Pore-scale models are also required to develop closure arguments for macroscopic models [17]. Furthermore, such models allow the investigation of flow and thermal dispersion effects.

The objective of this work is to extend to lower porosities the methodology described in our previous work on direct simulation of heat and fluid flow through open-cell foams [22]. In our previous work, we proposed a unit-cell body-centered cubic (bcc) geometry for representing open-cell foams. The detailed simulations on the foam unit cell were able to predict effective thermal conductivity, friction factor, and interstitial Nusselt number, which agreed with existing experimental measurements for $\varepsilon>0.94$. The present work examines alternative unit-cell representations for foams and identifies those which best capture experimental data on friction factor, Nusselt number, and effective thermal conductivity as a function of porosity. In the next section, we describe the foam geometry creation followed by a brief discussion of our mathematical and computational methodology [22]. Subsequently, we discuss the predictions obtained, and provide conclusions and remarks on future work.

\section{Foam Geometry}

In this work, we use the same methodology for geometry creation as discussed in Ref. [22]. The shape of the pore is assumed to be spherical and spheres of equal volume are arranged according to the following three lattice structures: (i) bcc, (ii) facecentered cubic (fcc), and (iii) A15 lattice, a geometry similar to Weaire-Phelan structure $[23,24]$. The periodic foam unit-cell geometry is obtained by subtracting the unit-cell cube from the spheres at the various lattice points, as shown in Fig. 2(a). The cross section of the foam ligaments is a set of convex triangles (Plateau borders), all of which meet at symmetric tetrahedral vertices [23]. It may be noted that there is a nonuniform distribution of metal mass along the length of the ligament with more mass accumulating at the vertices (nodes) resulting in a thinning at the center of the ligament as experimentally observed in foam samples by many authors (e.g., Ref. [25]). Figure 2(b) shows sample open-cell structures formed for three different lattice arrangements.

The distinguishing features of this approach are that (i) the geometry creation is simple, (ii) it captures many of the important features of real foams, and (iii) meshing of the geometry is easier compared to the approach adopted in Ref. [26] for computing pressure drop. 


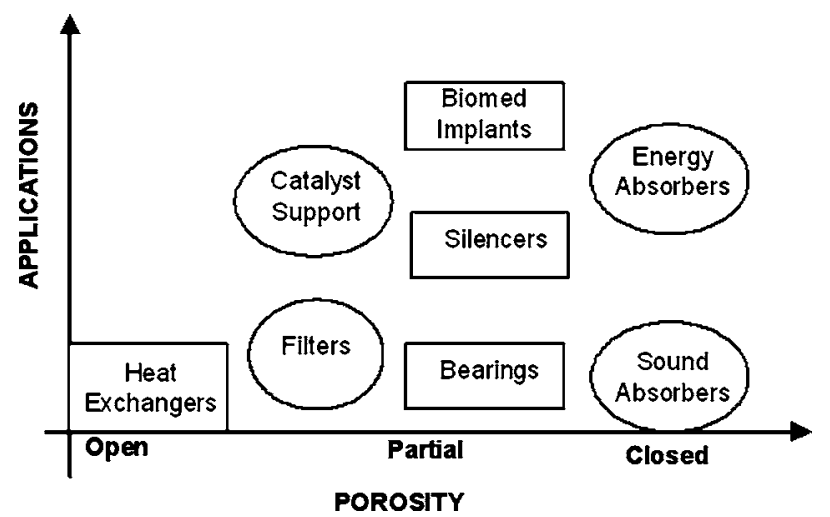

Fig. 1 Applications of porous cellular materials classified by the type of cellular geometry (adapted from Ref. [3])

\section{Mathematical Modeling}

Flow and Temperature Periodicity for Convective Flow and Heat Transfer Simulation. We first consider the formulation employed for computing flow and heat transfer through the pores; the formulation for computing effective thermal conductivity of foamfluid mixtures is given in a separate section. We consider a threedimensional periodic module with a constant translational vector (L) with respect to a flow direction ( $x$ axis), as shown in Fig. 3. For periodic boundaries, according to Ref. [27], the velocity and the pressure at any position $\mathbf{r}$ can be written as

$$
\begin{gathered}
u_{i}(\mathbf{r})=u_{i}(\mathbf{r}+\mathbf{L})=u_{i}(\mathbf{r}+2 \mathbf{L})=\cdots \\
P(\mathbf{r})-P(\mathbf{r}+\mathbf{L})=P(\mathbf{r}+\mathbf{L})-P(\mathbf{r}+2 \mathbf{L})=\cdots
\end{gathered}
$$

It should be noted that there may be other periodic boundaries in the module, but there is no net inflow through any of these boundaries. For flow through periodic unit cells, the pressure gradient can be divided into two components: (a) periodic component, $\partial \hat{p} / \partial x_{i}$, and (b) a linearly varying component, $\left(\partial \bar{p} / \partial x_{i}\right) \mathbf{e}_{L}$, such that $\partial P / \partial x_{i}=\left(\partial \bar{p} / \partial x_{i} \mathbf{e}_{L, i}\right)+\left(\partial \hat{p} / \partial x_{i}\right)$, where, $e_{L, i}$ is the $i$ th component of the unit vector in the direction $\mathbf{L}$.

A constant heat flux is imposed on the metal foam surfaces. For given heat-flux boundary conditions, the form of the temperature field becomes constant from module to module. Thus, the periodic condition for the temperature is given by

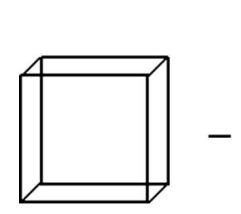

Cube

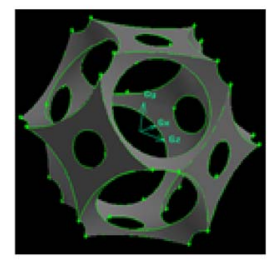

BCC

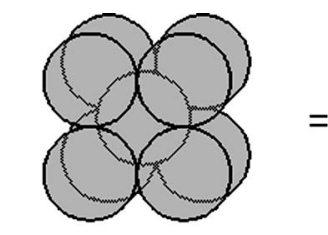

$\mathrm{BCC}$

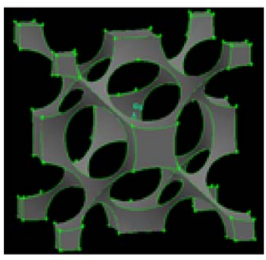

FCC

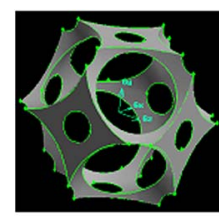

Final Geometry

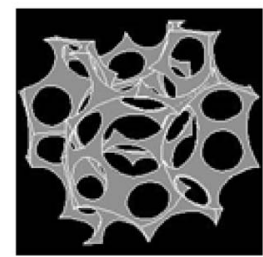

A15
Fig. 2 (a) Schematic representation of foam geometry creation and (b) sample images of foam geometry created for bcc, fcc, and A15 arrangements of spherical pores

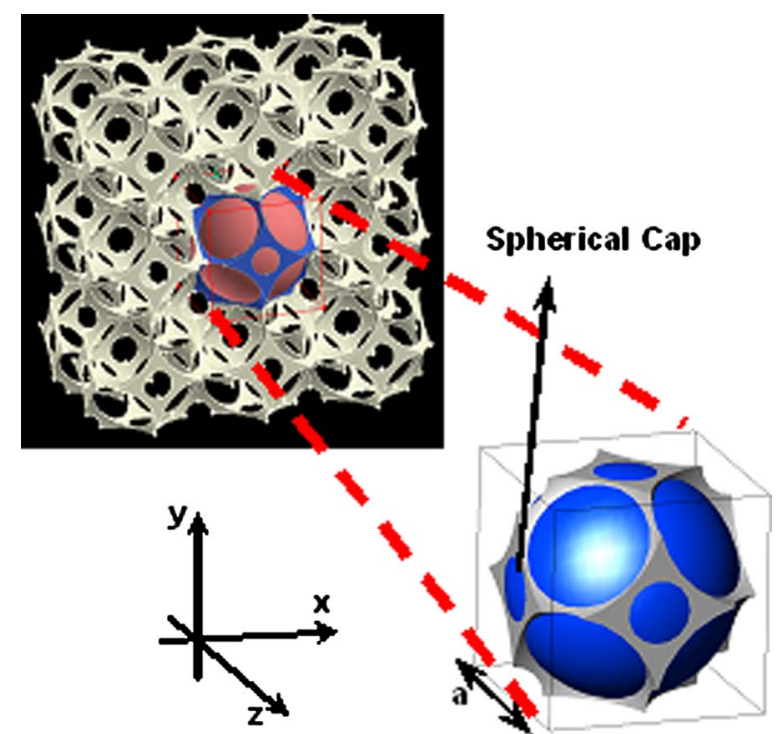

Fig. 3 Schematic illustration of a periodic domain

$T(\mathbf{r})-T_{b}(\mathbf{r})=T(\mathbf{r}+\mathbf{L})-T_{b}(\mathbf{r}+\mathbf{L})=T(\mathbf{r}+2 \mathbf{L})-T_{b}(\mathbf{r}+2 \mathbf{L})=\cdots$

Here, the bulk temperature $T_{b}$ is defined as

$$
T_{b}=\frac{\iint_{A}\left|u_{i} e_{L, i}\right| T d A}{\iint_{A}\left|u_{i} e_{L, i}\right| d A}
$$

where $A$ is the area of cross section.

Governing Equations. The governing flow and heat transfer equations for laminar, periodic, fully developed, incompressible, steady flow of a Newtonian fluid are $[27,28]$

$$
\begin{gathered}
\frac{\partial}{\partial x_{i}}\left(\rho u_{i}\right)=0 \\
\frac{\partial}{\partial x_{j}}\left(\rho u_{i} u_{j}\right)=-\frac{\partial \hat{p}}{\partial x_{i}}+\frac{\partial}{\partial x_{j}}\left(\mu \frac{\partial u_{i}}{\partial x_{j}}\right)_{i}-\frac{\partial \bar{p}}{\partial x_{i}} e_{L, i} \\
\frac{\partial}{\partial x_{i}}\left(\rho u_{i} C_{p} T\right)=\frac{\partial}{\partial x_{i}}\left(k \frac{\partial T}{\partial x_{i}}\right)
\end{gathered}
$$

The above equations are written assuming that the flow is thermally and hydrodynamically fully developed. Though the current study considers only the laminar flow condition, it can be extended to fully developed turbulent regimes. In Eq. (3), the terms involving $\partial^{2} / \partial x^{2}$ have been included to account for the large local streamwise gradients that may occur in periodically fully developed flows. The $\partial \bar{p} / \partial x_{i}$ term in Eq. (2) is assigned a priori, and controls the mass flow rate through the module, and hence, the pore Reynolds number. A no-slip boundary condition is imposed for the velocities on the bounding walls. As mentioned previously, a constant heat flux is specified for the energy equation on the foam surfaces. Details of the mathematical model are available in Refs. [22,27], while those on the numerical methods for periodic flow and heat transfer on unstructured meshes along with the implementation are outlined in Ref. [28].

The various periodic unit-cell geometries used in this work were created using the commercial software GAMBIT [29]. The geometry was discretized into three-dimensional finite volumes using hybrid (tetrahedral and hexahedral) elements in GAMBIT by specifying the minimum edge length. The finite volume mesh so 


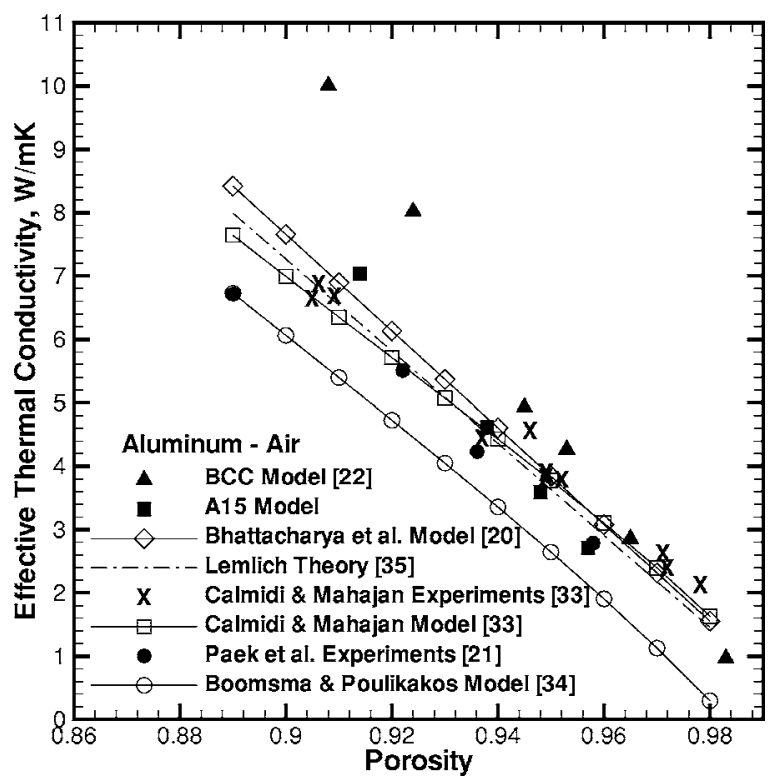

Fig. 4 Predicted effective thermal conductivity of an aluminum foam-air system for a range of porosities. Also plotted are available semiempirical models and experimental measurements.

created was exported to the commercial code FLUENT [30] for flow and heat transfer simulations. A second-order upwind scheme was used for the convective calculations. A colocated pressure-velocity formulation in conjunction with the SIMPLE algorithm was used for obtaining the velocity fields, and the linearized systems of equations solved using an algebraic multigrid algorithm. Details of the numerical method may be found in Ref. [31]. The calculations were terminated when the (scaled) residual [30] had dropped below $10^{-6}$ for all governing equations.

Grid-independence tests were performed using the procedures detailed in Ref. [22]. The calculations reported in this paper were performed using approximately 200,000 finite volumes.

\section{Results and Discussion}

Effective Thermal Conductivity. The numerical results for effective thermal conductivity for an aluminum-air system are considered first. The effective thermal conductivity is computed by numerically solving the conduction heat transfer through both the metal foam and the interstitial air on a single periodic module. A given heat-flux condition is not employed as above; instead, continuity of temperature and heat flux are employed as the interfacial conditions. Under the periodicity assumption, each module in the heat flow direction experiences an equal temperature drop. Computations are performed on a periodic module by imposing an arbitrary temperature drop $\Delta T$ across the periodic module along the heat flow direction, say, the $x$ axis. The resulting heat transfer rate at the periodic boundaries is used to obtain the effective thermal conductivity of the system using the relation

$$
k_{\mathrm{eff}}=\frac{-\int_{A_{p}} \mathbf{J} d \mathbf{A}}{\left(\Delta T A_{p} / \mathbf{L}\right)}
$$

where $\mathbf{J}$ is the diffusion flux vector at the periodic face, $d \mathbf{A}$ is the outward pointing elemental face area vector on the periodic face, and $A_{p}$ is the area of the periodic face. Calculations are performed using a modified version of the commercial code FLUENT [30]. Details of the mathematical model and numerical method are outlined in Refs. [22,32].

Figure 4 shows the calculated effective thermal conductivity for

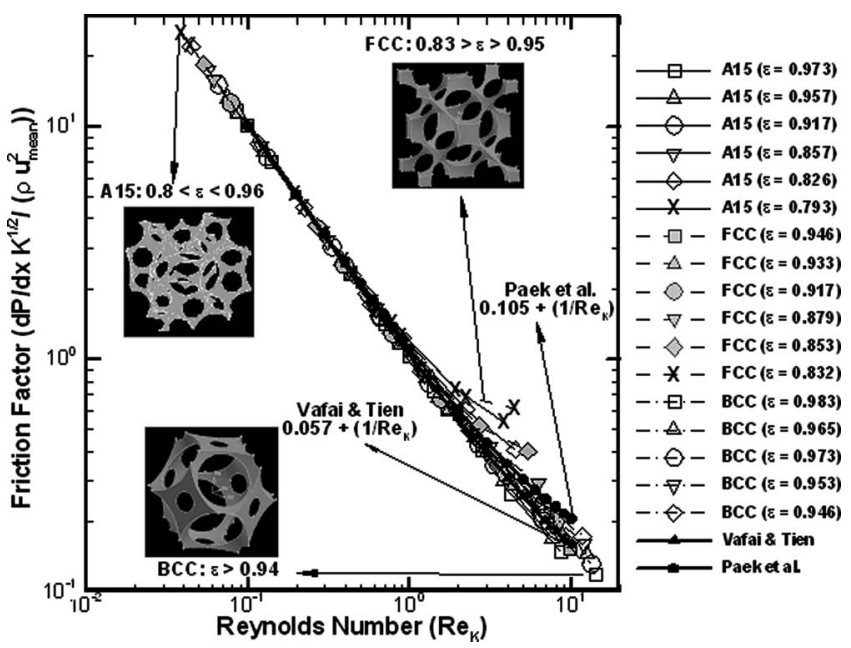

Fig. 5 Predicted friction factor as a function of modified Reynolds number $\left(\operatorname{Re}_{K}=\operatorname{Re}_{d}(V / D)\right)$. Results for the A15 lattice are plotted in red with symbols corresponding to the porosity range: $0.8<\varepsilon<0.96$. The fcc $(0.83<\varepsilon<0.95)$ and bcc $(\varepsilon>0.94)$ [22] results are shown in green and blue, respectively. Also, experimental correlations from Refs. $[21,36]$ are plotted.

an aluminum foam-air system as a function of foam porosity. Also plotted in Fig. 4 are the predictions from the available semiempirical models, and experimental measurements from the literature $[20,21,33,34]$. The available experimental effective thermal conductivity measurements for foams cover a porosity range of $0.89<\varepsilon<0.98$. Paek et al. [21] and Calmidi and Mahajan [33] reported experimental uncertainties of $12 \%$ and $3.6 \%$ for the effective thermal conductivity measurements, respectively. It should be noted that the bcc and A15 models used in the present work do not employ any arbitrary parameters to match the experiments. Also plotted is the theoretical result from Lemlich's original work on electrical conductivity of liquid foams [35]. Using a direct analogy between Ohm's law and Fourier's law, we adopt his results for effective thermal conductivity of metal foams $\left(k_{\mathrm{eff}}\right.$ $\left.=k_{s}(1-\varepsilon) / 3\right)$. Details of the Lemlich theory and its implications are discussed in Ref. [22]. It can be inferred from Fig. 4 that both A15 and bcc models compare well with the existing literature for high porosities. The bcc model, as discussed in Ref. [22], deviates from experiments for porosities below 0.94 because the foam geometry ceases to be open celled, and there is an accumulation of metal mass at the nodes. The predictions of the A15 model match experiments for porosities $\varepsilon>0.9$. However, there are no experimental data for porosities below 0.9, making validation difficult for high-density foams. In Fig. 4, the predictions from the fcc model are not plotted because the model underpredicted the experimental results by a factor of approximately 3 .

Pressure Drop and Nusselt Number. We now consider predictions of the pressure drop and Nusselt number for the fcc and A15 lattice models. The predictions of the bcc model [22] are also plotted for comparison. As noted above, a constant heat-flux boundary condition is imposed on the ligament walls for all the cases considered. Hence, heat conduction through the foam ligaments is not modeled. Constant temperature boundary conditions may be alternatively employed on the foam walls; the predicted Nusselt numbers under those conditions would differ somewhat from those predicted with the constant flux conditions employed in this study [27].

Figure 5 shows the results for the friction factor calculated for an aluminum foam-air system from the numerical simulations based on the fcc and A15 modules. Also plotted are the experimental correlations from Refs. [21,36]. Paek et al. reported the repeatability error for the measurement of pressure drop to be $3 \%$ 


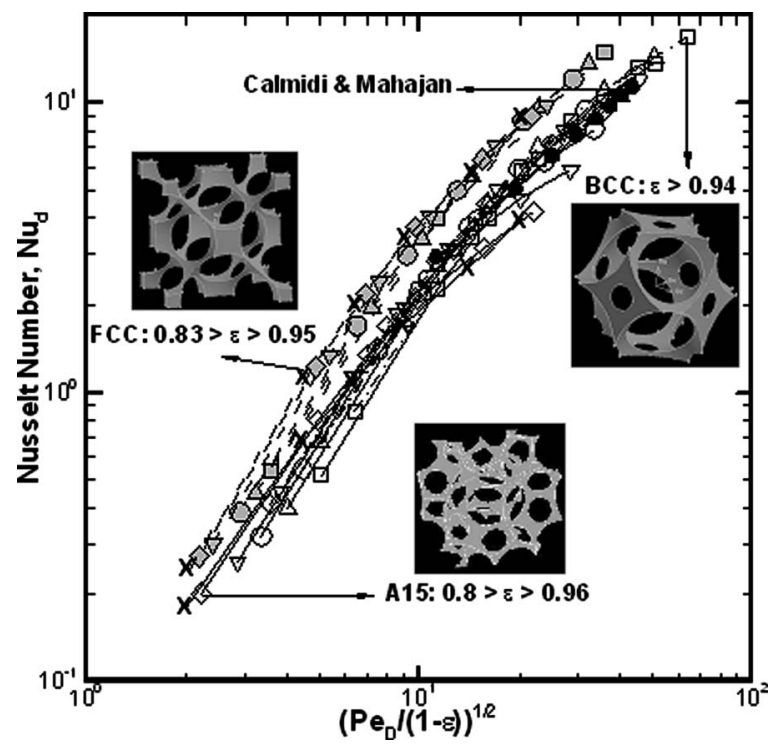

Fig. 6 Predicted Nusselt number as a function of square root of Peclet number. Results for the A15 lattice are plotted in red with symbols corresponding to the porosity range: $0.8<\varepsilon$ $<0.96$. The fcc $(0.83<\varepsilon<0.95)$ and bcc $(\varepsilon>0.94)$ [22] results are in green and blue, respectively. Please refer to Fig. 5 for the legend.

for foam samples of porosity $0.89<\varepsilon<0.97$. The porosity range considered by Vafai and Tien was $0.94<\varepsilon<0.97$. Furthermore, results for a bcc model from our previous work [22] are also plotted in the figure. The permeability of the foam was calculated using the expression $K=-\left(\mu u_{\text {mean }}\right) /\left(\partial \bar{p} / \partial x_{i}\right)$, where $u_{\text {mean }}$ was obtained for specified inlet mass flow rate in the Darcy regime ( $\mathrm{Re}$ $<10)$. The friction factor shown in Fig. 5 is defined as

$$
f=\frac{\left(-\partial \bar{p} / \partial x_{i}\right) \sqrt{K}}{\rho u_{\text {mean }}^{2}}
$$

The predicted friction factors from all the three models (bcc, fcc, and A15) compare well with the experimental correlations for porosities $\varepsilon>0.88$ and deviations for both the fcc and A15 are observed for porosities $<0.88$. It should be noted that the fcc model overpredicts the friction factor compared to the A15 model.

The Nusselt number for the foam was also calculated for the different cases considered and is defined as

$$
\mathrm{Nu}_{D}=\frac{h D}{k_{f}}=\frac{q^{\prime \prime} D}{k_{f}\left(\bar{T}_{s}-T_{b}\right)}
$$

In the above equation $\bar{T}_{s}$ is the averaged temperature of the foam. In Fig. 6, the predicted local Nusselt number for the fcc and A15 lattices is plotted as a function of $\left(\mathrm{Pe}_{D} /(1-\varepsilon)\right)^{1 / 2}$, where the modified Peclet number $\left(\mathrm{Pe}_{D}\right)$ is $\operatorname{Re}_{d} \operatorname{Pr}$. This $x$-axis scale is obtained by balancing the convective and axial diffusive fluxes. It may be noted that predictions from the A15 model compare well with those from the bcc model for $\varepsilon>0.86$ and with experimental correlation from Calmidi and Mahajan [22,33]. Also, plotted are experimental correlation from Calmidi and Mahajan [33]. For lower porosities $(<0.86)$, the local Nusselt number based on the permeability decreases due to the decrease in the permeability of the porous medium.

\section{Conclusions}

This paper presents an approach for the modeling of open-cell foam geometries for porosities $\varepsilon>0.8$. The distinguishing feature of this approach is that no adjustable geometric parameters were used to match the experiments, unlike in previous published work. The fcc and A15 unit-cell models, along with the bcc model considered previously [22], are shown to predict friction factor and Nusselt number values, which are in good agreement with available experimental and semianalytical results.

The bcc and A15 models also predict thermal conductivity reasonably well. The predictions from the A15 model compared well with available range of experimental thermal conductivity measurements $(\varepsilon>0.89)$ and bcc model predictions; the fcc model predictions for effective thermal conductivity showed greater deviations from the available measurements. Based on the results, there is a clear need for experimental results at foam porosities below 0.89 . For convective flow calculations, the predicted friction factor and local Nusselt number from both fcc and A15 models compare well with our previous (bcc) model and with available experimental results for $\varepsilon>0.86$. The accuracy of the pressure drop and Nusselt number predictions depends on how well the unit-cell representation captures the surface-to-volume ratio of the real foam. On the other hand, the effective thermal conductivity depends strongly on capturing the ligament resistance correctly, and would require an accurate representation of the ligament cross-sectional area-to-length ratio. An evaluation of unit-cell models along these lines would benefit from the characterization of actual foams for a range of porosities and manufacturing techniques.

\section{Acknowledgment}

Support for this work from industry members of the Cooling Technologies Research Center, a NSF Industry/University Cooperative Research Center (www.ecn.purdue.edu/CTRC), is gratefully acknowledged.

\section{Nomenclature}

$$
\begin{aligned}
a & =\text { edge length of the unit cell, } \mathrm{m} \\
A & =\text { area, } \mathrm{m}^{2} \\
C_{p} & =\text { specific heat capacity, } \mathrm{J} \mathrm{kg}^{-1} \mathrm{~K}^{-1} \\
D & =\text { diameter of the pore, } \mathrm{m} \\
\mathrm{Da} & =\text { Darcy number } \\
f & =\text { friction factor } \\
h & =\text { heat transfer coefficient, } \mathrm{W} \mathrm{m}^{-2} \mathrm{~K}^{-1} \\
\mathrm{~J} & =\text { diffusion flux vector, } \mathrm{m}^{2} \mathrm{~s}^{-1} \\
K & =\text { permeability, } \mathrm{m}^{2} \\
k & =\text { thermal conductivity, } \mathrm{W} \mathrm{m}^{-1} \mathrm{~K}^{-1} \\
L & =\text { length of the periodic } \text { module}, \mathrm{m} \\
\mathrm{Nu} & =\text { Nusselt number } \\
q^{\prime \prime} & =\text { heat flux, W } \mathrm{m}^{-2} \\
\mathrm{Pr} & =\text { Prandtl number } \\
\mathrm{Pe} & =\text { Peclet number } \\
R & =\text { radius of the pore, } \mathrm{m} \\
\mathrm{Re} & =\text { Reynolds number } \\
s & =\text { center-to-center distance, } \mathrm{m} \\
T & =\text { temperature, } \mathrm{K} \\
t & =\text { time, } \mathrm{s} \\
u, v & =\text { velocities along } x, y, z \text { directions, } \mathrm{m} \mathrm{s}^{-1} \\
V & =\text { volume, m } \\
x, y, z & =\text { Cartesian coordinates }
\end{aligned}
$$

Greek Symbols

$$
\begin{aligned}
\alpha & =\text { thermal diffusivity, } \mathrm{m}^{2} \mathrm{~s}^{-1} \\
\varepsilon & =\text { porosity } \\
\mu & =\text { dynamic viscosity, } \mathrm{kg} \mathrm{m}^{-1} \mathrm{~s}^{-1} \\
\rho & =\text { density, } \mathrm{kg} \mathrm{m}^{-3}
\end{aligned}
$$

Superscript

$$
\text { - = average or mean }
$$

Subscripts

$$
\begin{aligned}
b & =\text { bulk } \\
\mathrm{bc} & =\text { body center }
\end{aligned}
$$




$$
\begin{aligned}
D & =\text { Darcian } \\
\text { eff } & =\text { effective } \\
f & =\text { fluid } \\
\text { in } & =\text { inlet } \\
\text { int } & =\text { intersection } \\
K & =\text { permeability } \\
s & =\text { solid } \\
\mathrm{sa} & =\text { surface area } \\
\mathrm{sc} & =\text { spherical cap }
\end{aligned}
$$

\section{References}

[1] Gibson, L. J., and Ashby, M. F., 1999, Cellular Solids: Structure and Properties, Cambridge University Press, New York

[2] Ashby, M. F., Evans, A., Fleck, N. A., Gibson, L. J., Hutchinson, J. W., and Wadley, H. J. G., 2000, Metal Foams: A Design Guide, ButterworthHeinemann, Boston.

[3] Banhart, J., 2001, "Manufacture, Characterization and Application of Cellular Metals and Metal Foams," Prog. Mater. Sci., 46, pp. 559-632.

[4] Lakes, R. S., 1987, "Foam Structures With a Negative Poisson's Ratio," Science, 235, pp. 1038-1040.

[5] Chan, N., and Evans, K. E., 1997, "Fabrication Methods for Auxetic Foams," J. Mater. Chem., 32, pp. 5945-5953.

[6] Preciado, J. A., Cohen, S., Skandakumaran, P., and Rubinsky, B., 2003, "Utilization of Directional Freezing for the Construction of Tissue Engineering Scaffolds," HTD (Am. Soc. Mech. Eng.), 374, pp. 439-442.

[7] Freyman, T. M., Yannas, I. V., and Gibson, L. J., 2001, "Cellular Materials as Porous Scaffolds for Tissue Engineering,” Prog. Mater. Sci., 46, pp. 273-282.

[8] Heung, L. K., 2002, "Design of Metal Hydride Vessels for Processing Tritium," Sixth International Conference on Tritium Science and Technology, pp. $1-11$.

[9] Peng, Y., and Richardson, J. T., 2004, "Properties of Ceramic Foam Catalyst Supports: One-Dimensional and Two-Dimensional Heat Transfer Correlations," Appl. Catal., A, 266, pp. 235-244.

[10] Fend, T., Hoffschmidt, B., Pitz-Paal, R., Reutter, O., and Rietbrock, R., 2004, Energy, 29, pp. 823-833.

[11] Montillet, A., Comiti, J., and Legrand, J., 1993, "Application of Metallic Foams in Electrochemical Reactors of Filter-Press Type Part I: Flow Characterization," J. Appl. Electrochem., 23, pp. 1045-1050.

[12] Kamiuto, K., Andou, J., Miyanaga, T., and Taniyama, S., 2005, "Mist Transpiration Cooling System Using Open-Cellular Porous Materials," J. Thermophys. Heat Transfer, 19, pp. 250-251.

[13] Krishnan, S., Garimella, S. V., Chrysler, G. M., and Mahajan, R. V., 2005 "Towards a Thermal Moore's Law," IEEE Trans. Adv. Packag., 30, pp. 462 474.

[14] Ozmat, B., Leyda, B., and Benson, B., 2004, "Thermal Applications of OpenCell Metal Foams," Mater. Manuf. Processes, 19, pp. 839-862.

[15] Boomsma, K., Poulikakos, D., and Zwick, F., 2003, "Metal Foams as Compact High Performance Heat Exchangers," Mech. Mater., 35, pp. 1161-1176.

[16] Klein, J., Gilchrist, G., Karanik, J., Arcas, N., Yurman, R., Whiteside, J., Shields, B., and Bartilucci, T., 2003, "Thermal Management of Airbourne
Early Warning and Electronic Warfare Systems Using Foam Metal Fins," International Electronic Packaging Technical Conference and Exhibition (IP ACK'03), Paper No. IPACK2003-35187.

[17] Krishnan, S., Murthy, J. Y., and Garimella, S. V., 2004, "A Two-Temperature Model for the Analysis of Passive Thermal Control Systems," ASME J. Heat Transfer, 126, pp. 628-637.

[18] Krishnan, S., Murthy, J. Y., and Garimella, S. V., 2005, "A Two-Temperature Model for Solid-Liquid Phase Change in Metal Foams," ASME J. Heat Transfer, 127, pp. 995-1004.

[19] DuPlessis, P., Montillet, A., Comiti, J., and Legrand, J., 1994, "Pressure Drop Prediction for Flow Through High Porosity Metallic Foams," Chem. Eng. Sci. 49, pp. 3545-3553.

[20] Bhattacharya, A., Calmidi, A. A., and Mahajan, R. L., 2002, "Thermophysical Properties of High Porosity Metal Foams,” Int. J. Heat Mass Transfer, 45, pp. 1017-1031.

[21] Paek, J. W., Kang, B. H., Kim, S. Y., and Hyun, J. M., 2000, "Effective Thermal Conductivity and Permeability of Aluminum Foam Materials," Int. J. Thermophys., 21, pp. 453-464

[22] Krishnan, S., Murthy, J. Y., and Garimella, S. V., 2006, "Direct Simulation of Transport in Open-Cell Metal Foam," ASME J. Heat Transfer, 128, pp. 793799

[23] Phelan, R., Weaire, D., and Brakke, K., 1995, “Computation of Equilibrium Foam Structures Using the Surface Evolver," Exp. Math., 4, pp. 181-192.

[24] Weaire, D., 2001, "A Philomorph Looks at Foam," Proceedings of the American Philosophical Society, Vol. 145, pp. 564-574.

[25] Dharmasena, K. P., and Wadley, H. N. G., 2002, "Electrical Conductivity of Open-Cell Metal Foams," J. Mater. Res., 17, pp. 625-631.

[26] Boomsma, K., Poulikakos, D., and Ventikos, Y., 2003, "Simulations of Flow Through Open Cell Foams Using an Idealized Periodic Cell Structure," Int. J. Heat Fluid Flow, 24, pp. 825-834.

[27] Patankar, S. V., Liu, C. H., and Sparrow, E. M., 1977, "Fully Developed Flow and Heat Transfer in Ducts Having Streamwise-Periodic Variations of CrossSectional Area," ASME J. Heat Transfer, 99, pp. 180-186.

[28] Murthy, J. Y., and Mathur, S., 1997, "Periodic Flow and Heat Transfer Using Unstructured Meshes," Int. J. Numer. Methods Fluids, 25, pp. 659-677.

[29] Fluent Inc., User's Guide for GAMBIT 2.0, 2002.

[30] Fluent Inc., User's Guide for FLUENT 6.0, 2002.

[31] Mathur, S. R., and Murthy, J. Y., 1997, "A Pressure-Based Method for Unstructured Meshes," Numer. Heat Transfer, Part B, 31, pp. 195-216.

[32] Kumar, S., and Murthy, J. Y., 2005, "A Numerical Technique for Computing Effective Thermal Conductivity of Fluid-Particle Mixtures," Numer. Heat Transfer, Part B, 47, pp. 555-572.

[33] Calmidi, V. V., and Mahajan, R. L., 1999, "The Effective Thermal Conductivity of High Porosity Fibrous Metal Foams," ASME J. Heat Transfer, 121, pp. 466-471.

[34] Boomsma, K., and Poulikakos, D., 2001, "On the Effective Thermal Conductivity of a Three-Dimensionally Structured Fluid-Saturated Metal Foam," Int J. Heat Mass Transfer, 44, pp. 827-836.

[35] Lemlich, R., 1978, "A Theory for the Limiting Conductivity of Polyhedra Foam at Low Density," J. Colloid Interface Sci., 64, pp. 107-110.

[36] Vafai, K., and Tien, C. L., 1982, "Boundary and Inertia Effects on Convective Mass Transfer in Porous Media," Int. J. Heat Mass Transfer, 25, pp. 11831190 